\section{NEEDLESTICK INJURIES AMONG SANITATION WORKERS IN MEXICO CITY}

Sanitation workers (SW) are exposed to a wide range of biological, chemical, and physical hazards. Needlestick injuries constitute a recognized physical hazard, but few studies have been conducted to document the magnitude of the problem among SW. Contaminated sharp objects can transmit bloodborne pathogens, such as human immunodeficiency (HIV), hepatitis B (HBV), and hepatitis C (HCV) viruses. Needles and syringes contaminated with $\mathrm{HBV}$ and $\mathrm{HCV}$ have been found in public recreational areas in South London, United Kingdom (1). Although transmission of such pathogens to sanitation workers and community members has not been demonstrated, there is the potential for infection. We were not aware of any prior study among SW in a developing country. Given this lack of information, we conducted a survey of needlestick and sharps injuries among SW in Mexico City.

In July-August 2003, a non-random, convenience sample of $69 \mathrm{SW}$ was selected from 13 of the 16 Mexico City districts by finding and approaching sanitation vehicles during their daily rounds. Workers were identified and interviewed on the street, while working or during a break. Questionnaires determined the frequency of sustaining a needlestick injury at work, finding syringes with regular waste, vaccination status, and health care-seeking practices. Epi $\mathrm{Info}^{\mathrm{TM}}$ Version 3.3.2 (Centers for Disease Control and Prevention, Atlanta, Georgia, United States of America) was used for data entry, descriptive epidemiology, and analysis.

The study was exempted from human subjects review by the Institutional Review Board of the Centers for Disease Control and Prevention because no personal identifiers were collected and no questions were considered sensitive. The study was approved by the Research Oversight Committee of the National Immunization Council, Mexico City, Mexico.

Although the total sample of SW was 69, not all responded to every question; thus denominators vary among these results. Fifty (82\%) of 61 respondents were employed by the municipal government. Twenty-five $(38 \%)$ of 66 respondents worked on a truck; the remainder transferred waste from residences or small businesses into large barrels that were then emptied into trucks. Of the $69 \mathrm{SW}$ interviewed, 13 $(34 \%)$ reported 22 needlesticks (median $=1$; range: $1-5)$ during the previous 12 months. Fourteen of these needlesticks were reported by $7(10 \%)$ workers in the previous month. Sixty-six (96\%) of the 69 SW had seen needles and syringes within waste at some time in the past, and $56(81 \%)$ had seen them during the previous week. Fifty-five percent of SW saw syringes/needles more than five times in the previous week. Most sightings $(81 \%)$ of needles and syringes occurred in residential areas. Only $6(11 \%)$ of 55 SW reported that they always wore gloves at work and only 3 workers (4\%) reported being vaccinated against HBV. SW in Mexico City generally are not encouraged to receive hepatitis $B$ vaccine, nor is the vaccine provided free of charge. When the 31 workers who reported ever being stuck by a needle were asked what they did following a needlestick injury, the responses were: $13(42 \%)$ washed or disinfected the wound, $6(19 \%)$ pushed the "bad blood" out or put lime on the wound, $4(13 \%)$ sought medical care, and $8(26 \%)$ did nothing.

Needlesticks occurred frequently among the sanitation workers interviewed in Mexico City, and their sightings of used hypodermic needles in trash occurred even more often. Assuming the findings in this non-random, convenience sample reflect the experience of other SW in Mexico City, the high frequency of needlesticks, infrequent use of gloves for personal protection, and poor hepatitis B immunization coverage would put this occupational group at high risk of acquiring bloodborne infections.

Although there are several studies elsewhere on occupational injuries among SW, few address the specific problem of needlestick and sharps injuries. A study of community-acquired needlestick injuries in New South Wales, Australia, found that cleaners were the non-healthcare occupational group most frequently seen at urban emergency departments (2). A 1989 study among 940 waste industry workers from Washington State in the United States found that $6 \%$ suffered needlesticks with hypodermic needles while working, and 90\% reported seeing used needles and syringes discarded with regular waste (3).

In another study that examined health records of New York City Sanitation Department waste workers, needlesticks were reported from needles in waste collected from hospitals and physician and dental offices, as well as discards from drug addicts (4). In the present study, the large majority of the needles and syringes were sighted in residential areas. Most likely, these were discarded by the residents themselves, given the easy access (a physician's prescription is not required) and low-cost of hypodermic needles in Mexico City pharmacies. A general preference for injectable medications may also contribute to the greater use of disposable needles and syringes by Mexico City residents (5).

Discarded sharps may be contaminated with bloodborne pathogens as demonstrated by the presence of $\mathrm{HBV}$ or $\mathrm{HCV}$ in $9.4 \%$ of discarded syringes in city parks in London (6). Although the risk of infection 
with bloodborne pathogens is considered to be low in community settings, their occurrence has been reported in a few studies (1).

This study has a number of limitations. The convenience sample of SW who participated in this study may not be representative of all such workers in Mexico City. Querying for events in the previous 12 months may result in recall bias, either over- or underestimation of events and subjective responses. Further, these interviews during working hours resulted in missing response rates of up to $21 \%$ to some questions, especially those requiring extensive recall.

The presence of needles and syringes with regular waste and the resulting needlestick injuries are due to improper disposal of sharps by users. Health care providers and pharmacists could educate community members who receive injections on proper disposal of sharps. SW ought to use personal protective gear, be vaccinated against $\mathrm{HBV}$, and receive post-exposure prophylaxis in accordance with public health guidelines (7-9) as a means to reduce the number of occupational sharps injuries and their consequences among these workers.

Brenda Thompson University of Nebraska Medical Center Omaha, Nebraska, United States of America Pedro L. Moro Immunization Safety Office Division Of Healthcare Quality Promotion, Centers for Disease Control and Prevention (CDC) Atlanta, Georgia, United States of America psm9@cdc.gov Kattrina Hancy

Farmworker Justice Washington DC, United States of America Ismael R. Ortega-Sánchez Division of Viral Diseases, National Center for Immunization and Respiratory Diseases, CDC Atlanta, Georgia, United States of America José I. Santos-Preciado Hospital Infantil de México Federico Gómez México D.F, México
Carlos Franco-Paredes

Emory University School of Medicine Atlanta, Georgia, United States of America

Bruce G. Weniger

Division of Bacterial Diseases,

National Center for Immunization and Respiratory Diseases, CDC

Atlanta, Georgia, United States of America

Robert T. Chen

Division of HIV / AIDS Prevention National Center for HIV, Viral Hepatitis, STD, and TB Prevention, CDC Atlanta, Georgia, United States of America

\section{REFERENCES}

1. Nyri P, Leung T, Zuckerman MA. Sharps discarded in inner city parks and playgrounds-risk of bloodborne virus exposure. Commun Dis Public Health. 2004;7(4):287-8.

2. O'Leary FM, Green TC. Community acquired needlestick injuries in non-health care workers presenting to an urban emergency department. Emerg Med. 2003;15(5-6):434-40.

3. Turnberg WL, Frost F. Survey of occupational exposure of waste industry workers to infectious waste in Washington State. Am J Public Health. 1990;80(10):1262-4.

4. Lawitts S. Needle sightings and on-the-job needle-stick injuries among New York City Department of Sanitation Workers. J Am Pharm Assoc. 2002;42(6 Suppl 2):S92-3.

5. Pylypa J. Self-medication practices in two California Mexican communities. J Immigr Health. 2001;3(2):59-75.

6. Haber P, Young M, Dorrington L, Jones A, Kaldor J, De Kanzow $\mathrm{S}$, Rawlinson $\mathrm{W}$. Transmission of hepatitis $\mathrm{C}$ virus by needlestick injury in community settings. J Gastro Hepatol, 2007;22(11): 1882-5.

7. World Health Organization. Health and safety practices for health-care personnel and waste workers. In: Pruss A, Giroult E, Rushbrook P, eds. Safe management of wastes from healthcare activities. Geneva. WHO; 1999:140-4. Available at: http:/ / www.who.int/water_sanitation_health/medicalwaste/140to 144.pdf. Accessed 28 May 2009.

8. Wong ES, Stotka JL, Chinchilli VM, Williams DS, Stuart G, Markowitz SM. Are universal precautions effective in reducing the number of occupational exposures among health care workers? JAMA 1991;265(9):1123-8.

9. Department of Health. Health Technical Memorandum 07-01: safe management of healthcare waste. Available at: http:// www.dh.gov.uk/en/Publicationsandstatistics/Publications/ PublicationsPolicyAndGuidance/DH_063274. Accessed 9 July 2009. 